Original Research Paper

\title{
Comprehending the Environmental and Household Determinants of Jaundice Prevalence in Western Nepal
}

\author{
${ }^{1}$ Uttam Paudel* and ${ }^{2}$ Krishna Prasad Pant \\ ${ }^{1}$ PhD Candidate of Environmental Health Economics, Tribhuvan University, Nepal \\ ${ }^{2}$ Environmental Economist, Kathmandu University, Kathmandu, Nepal
}

\author{
Article history \\ Received: 23-08-2019 \\ Revised: $18-01-2020$ \\ Accepted: $17-02-2020$ \\ Corresponding Author: \\ Uttam Paudel \\ $\mathrm{PhD}$ Candidate of \\ Environmental Health \\ Economics, Tribhuvan \\ University, Nepal \\ Email: uuupaudel22@gmail.com
}

\begin{abstract}
Persuasive but scanty evidences from literature claim that climate change is linked with neonatal and adult Jaundice prevalence, especially in underdeveloped countries. This paper explored major environmental and household characteristics attributable to Jaundice prevalence in western Nepal. Cross-sectional dataset obtained from household survey are employed to obtain the results from probit regression model, adequately leveled with an econometric theory. The results reveal that heat wave, warmer winter temperature and sporadic rain are the potential environmental factors influencing Jaundice to be urgently addressed by the national agenda of adaptation program in western Nepal. Control in family size, construction of flushed toilet in each household, proper disposal of solid waste released from kitchen and setting habit of drinking boiled water are found the major household adaptation practices to be advocated along with environmental protection campaign for control of Jaundice prevalence in western Nepal. Henceforth, the recommendations are made for policymakers accordingly.
\end{abstract}

Keywords: Western Nepal, Environment, Jaundice Prevalence, Climate Change, Household Attributes, Health

\section{Introduction}

Jaundice is a yellowing of skin, whites of eyes and body fluids caused by an increase in amount of bilirubin in blood produced from breakdown of heme, primarily from hemoglobin and red blood cells (Kumar and Bhowmik, 2011). Jaundice is not a disease but considered as a sign of an underlying disease process (Raguraman et al., 2017). In healthy people, liver metabolizes and excretes the bilirubin in the form of bile, however Jaundice may result if there is a disruption in this normal metabolism of bilirubin (Slusher et al., 2017). However, this biological concept embraces internal metabolism in human body and precludes other physical endogenous and exogenous factors causing the Jaundice prevalence in rural communities of developing countries.

Environmental factors such as air, water and climate change etc., are seemed responsible to affect Jaundice occurrence and its spread. Waterlogging and insanitary conditions leading to Jaundice, gastrointestinal disorders and cholera are common in developing countries (Mitra et al., 2015). In Bangladesh, number of health problems like skin diseases, Jaundice and Hepatitis-B are associated with extreme weather events (Kabir and Arafat, 2018). Another research strongly explored that Jaundice, diarrhea and malnutrition are water pollution related diseases (Raguraman et al., 2017). A review paper concluded that raising temperature influences the Jaundice prevalence, with advocating for the appropriate adaptation strategy (Pires et al., 2016). Sever neonatal Jaundice incidence in South Asia is reported about 251 per 10000 live births, which is the second highest after African region (Slusher et al., 2017). Finding factors attributable to Jaundice occurrence, mostly ignored and poorly covered by few international studies but high prevalence among rural people of developing countries, could be therefore an important research area.

Plenty of literatures are accessible regarding the frequency of neo-natal Jaundice linking with breast feeding (Chen et al., 2011) and other child care activities which is less concerned with environmental changes and household characteristics. The major environmental factors that may cause Jaundice occurrence and its exacerbation might have multiple spillover effects to cause human mortality and morbidity. A review paper covering high flood prone areas around the world claims 
that increase in severity and frequency of flooding caused by climate change is responsible for Jaundice outbreak (Dayrit et al., 2018). El'Nino southern oscillation is associated with increases in the occurrence of certain vector-borne and waterborne diseases promoting Jaundice (Azavedo et al., 1988).

A study from eastern Nepal reported that people living around highly flooded and drought-prone areas of Koshi Basin are prone to diarrhea and Jaundice (Chen, 1999). Similarly, an empirical study concerning environment-disease relationship claimed that disease prevalence in western Nepal is directly associated with environmental changes (Paudel and Pant, 2018). A recent review paper (Paudel, 2018a) based on the literature stated that water communicable disease including Jaundice are directly related with environmental attributes. An increasing trend of Jaundice occurrence is stated by annual report of Ministry of Health and Population (unpublished), in Nepal since 2005, however the Jaundice appearance among the children less than 2 months is almost constant. These evidences show that Nepal is equally prone with environmental changes and Jaundice occurrence.

None of the evidence has yet found explored the environment and Jaundice relationship in Nepal. A handful international set of literature are available for the representation of developing countries. From the review of all available national and international literature, none of the papers has established the relationship between the environmental factors and Jaundice occurrence in Nepal (Paudel, 2018a). Therefore, this paper explores possible environmental factors and household characteristics that are mainly responsible to influence the Jaundice occurrence at household level in western Nepal.

\section{Materials and Methods}

\section{Research Design}

Primary data with both the qualitative and quantitative approaches were employed in this study to explore the environmental and household behavioral factors influencing Jaundice among the rural community in western Nepal through cross-sectional analytical design. A semistructured household survey questionnaire was finalized after a reconnaissance survey, pretest and expert's suggestions. A total of 420 households were interviewed. The household data were complemented with record reviews, focus group discussions and in-depth interviews with health professionals and community people using semi-structured questionnaire. Collected data were entered into the SPSS and transferred to STATA for the analysis. With reference to some literatures, econometric theories are rigorously employed for the results of the logit/probit model in the formulation of environmentaldisease relationships. Moreover, results are thoroughly discussed and conclusion is drawn from the major and minor findings of the entire research.

\section{Description of the Study Areas, Setting and Designing}

This paper is the product of further econometric analysis of a descriptive analysis made in the previous paper based on the same data source. Therefore, the study areas and the study setting and design are exactly the same as the previous study (Paudel et al., 2018). This research covers the identification of environmental and household level factors influencing Jaundice occurrence across western Nepal.

\section{Empirical Methods for Analysis}

Initially, to understand the impact of environmental change on the diseases, an unknown functional relationship is considered as:

$Q=f(E, X)$

Relation (1) links vectors of environmental factors $(E)$ and household behavioral variables $(X)$ to outcomes (Jaundice occurrence), $Q . E$ includes knowledge and perception a bout environmental changes, natural disasters due to environmental change. A linearized version of the above model is:

$Q=\alpha+\beta E+\gamma X+\varepsilon$

Here, $Q$ is categorical variable, setting code 1 for Jaundice occurrence in particular household and code 0 for not. So, probit and logistic regression models are used to find the probable environmental and other factors encouraging Jaundice prevalence. The vector $X$ typically includes several controls also. Then, most pertinent and some literature-based variables possibly affecting the Jaundice were identified and hypothesized (Table 1) making dummy where necessary and made free from co-linearity with the help of zero order correlation matrix. More modeling aspect is explained in the next section.

\section{Econometric Treatment}

Focusing on the probit analysis in this study, it is better to concoct a theory for probit analysis. Since the dependent variable as a binary random variable, a logistic regression model seems apposite for the concoction of the results. Let $y_{t}$ be the binary random variable taking the value of 1 with probability $P_{t}$, can be written as:

$P_{t}=\frac{\exp \left(\beta^{\prime} x_{t}+v_{t}\right)}{1+\exp \left(\beta^{\prime} x_{t}+v_{t}\right)}$ 
Table 1: Description of hypothesized variables affecting jaundice

\begin{tabular}{lllll}
\hline Variable's name & Description & Expected sign & Mean & Standard deviation \\
\hline E02Winter_Temp & Increasing winter temperature & $+\mathrm{ve}$ & 0.635 & 0.481 \\
F01Spo_Ra & Untimely rain & $+\mathrm{ve}$ & 0.226 & 0.418 \\
J02HotAir & Hot wave & $+\mathrm{ve}$ & 0.721 & 0.269 \\
A12Fam_Size & Family Size & $+\mathrm{ve}$ & 6.540 & 3.486 \\
B07AInc_Source & Agriculture the main source of income & $+\mathrm{ve}$ & 0.733 & 0.484 \\
B06ToiletType & Non-flushed toilet type & $+\mathrm{ve}$ & 0.650 & 0.477 \\
C06Vetenary_Dis & Axcess of veterinary centers & $-\mathrm{ve}$ & 5.103 & 6.516 \\
D01Mem_Mic_Fin & Membership in microfinance & $-\mathrm{ve}$ & 0.242 & 0.429 \\
D05Dis_Motorway & Distance to motorway & $+\mathrm{ve}$ & 4.139 & 4.501 \\
D06Dis_healthpost & Distance to health post & $+\mathrm{ve}$ & 2.773 & 1.982 \\
L07SW_DisKit & Proper management of solid waste at home & $-\mathrm{ve}$ & 0.783 & 0.412 \\
L09Wat_Pref & Boiled water preference & $-\mathrm{ve}$ & 0.376 & 0.400
\end{tabular}

and 0 with probability $\left(1-P_{t}\right)$; where, $\beta$ the vector of unknown parameter, $x_{t}$ is a vector of known constant and $v_{t}$ is a random variables with zero mean and constant variance $\sigma^{2}$. If it is assumed that there are $n_{t}$ independent observations on $y_{t}$ denoted as $y_{t}(1), y_{t}(2), \ldots \ldots, y_{t}\left(n_{t}\right)$ and we write:

$$
P_{t}=\frac{1}{n_{t}} \sum_{t=1}^{t=n_{t}} y_{t}(i)
$$

This model is different from the standard logit model only in that $v_{t}$ is included in the right hand side of (1). Here, $v_{t}$ is assumed to have constant variance and nonzero mean. Then the probability function takes the form:

$$
Z_{t}=\beta^{\prime}\left(x_{t}+v_{t}+u_{t}\right)
$$

where, $Z_{t}=\log \left(\frac{P_{t}}{1-P_{t-1}}\right)$ and $u_{t}=\frac{p_{t}-P_{t}}{p_{t}\left(1-P_{t}\right)}$.

It is important to note that once $v_{t}$ is realized the same, $P_{t}$ applies to every observation $y_{t}(i)=1,2, \ldots, n_{t}$ Therefore, sometimes $P_{t}$ can be taken as unknown parameter rather than random variables with zero mean and variance equal to $\frac{1}{\left[n_{t} p_{t}\left(1-P_{t}\right)\right]}$ and, $u_{t}$ and $v_{t}$ are uncorrelated because the conditional mean of $u_{t}$, given $v_{t}$, is zero. Thus, the Equation (3) can be treated as logit regression equation with heterokedastic residuals, such that the variance of the $i^{\text {th }}$ residual is equal to estimated value of coefficients.

Applying the above model on disease prevalence and following Paudel et al. (2020), a binary dependent variable, let $P_{x}$ be the probability of changing disease prevalence due to the possible change in explanatory (exposure) variables $x$ and taking the value of 1 with given probability of changing disease prevalence. The probability of increasing or decreasing the diseases prevalence can be expressed as:
$P_{x}=P\left(\left.D\right|_{X=x}\right)=\frac{1}{1+e^{(-a+b x)}}$

Therefore, to identify the determinants of the disease prevalence, the final logistic regression equation for this study then takes the form:

$Y_{i t} \beta_{0}+\beta_{j} E_{i j t}+\beta_{k} X_{i k t}+\beta_{l} B_{i l t}+e_{i t}$

Where:

$Y_{i t}$ : Disease prevalence of $i^{\text {th }}$ household at time interval $t$

$\beta_{0}$ : Constant coefficient

$\beta_{j}$ : Vector of coefficients for environmental variables

$E_{i j t}$ : Vector of environmental variables at time $t$

$\beta_{k}$ : Vector of coefficients of demographic and socioeconomic variables

$X_{i k t}$ : Vectors of control (demographic and socioeconomic) variables

$\beta_{l}$ : Vector of coefficients of household behavioral variables

$B_{i l t}$ : Vector of household behavioral variables

$e_{i t}$ : Random error term

This general model (7) is applicable for the particular probit regression on Jaundice rampant with all the independent variables.

\section{Results}

\section{Descriptive Results}

Of the total respondents in the study, $55 \%$ were males. More than $60 \%$ of Jaundice cases are on males. The average household size in the study area is 6.5. Agriculture is the main occupation whose household size is bigger than the national average. Interestingly, almost $69 \%$ of respondents were illiterate or with primary level of education. Remarkably; almost $82 \%$ of households 
suffering from Jaundice responded change in climate condition, air pollution, biodiversity loss and change in water resources in the community. Above $83 \%$ of households that faced Jaundice have no water treatment devices and $11 \%$ have no toilet at home.

Households with simple toilet with big family size are seemed to have the highest level of Jaundice occurrence (Fig. 1). Therefore, flush toilet is seemed better to be protective from the chance of having the Jaundice occurrence, irrespective to the size of family.

Similarly, males are suffered much from Jaundice, though their average educational level is comparatively higher than that of females. This result reveals that males with Jaundice incidence are seemed higher average education level than their counterparts facing no any Jaundice cases at households (Fig. 2), meaning that Jaundice encouraging factors might be other than education level of household head.

Besides, households with unmarried people with age nearly 60 are afflicted a lot from Jaundice in western Nepal (Fig. 3), might be because of unmanaged males may have poor food and sanitation as normally the responsibilities of household food and sanitation are on females by culture in Nepal.

\section{Results of Econometric Analysis}

After obtaining zero order correlation coefficients among the selected variables, they were found to be free from any multi-colinearity problem. From the iterative process to identify the potential environmental factors, perception of increasing winter temperature, sporadic rain and heat wave (in model I) are found significant to explain the variations of Jaundice prevalence in western Nepal (Table 2). Being consistent with some international studies
(Raguraman et al., 2017; Drew et al., 1978) and experiences; untimely rain and heat waves in summer are likely to support Jaundice prevalence, while warm winter temperature is less likely to increase the probability chance of Jaundice prevalence. More precisely, decreasing the temperature from existing level leads to decrease the Jaundice cases at the rate of 0.6 (95\% C.I. $=$ 0.336-1.073). While, increasing the sporadic rain leads to an increase in the Jaundice prevalence by 1.97 (95\% C.I. $=1.139-3.413)$. These results are parallel with some limited studies (Mitra et al., 2015; Kabir et al., 2017).

Similarly, larger the family size, higher is the probability chance of having Jaundice among family members. More accurately, one more unit of member in the family leads to increase in Jaundice occurrence by 1.08 (95\% C.I. $=1.017-1.154)$. This is because with large family size, many family members may get exposed to Jaundice risks and further, family head might not be able to timely cure of all family members due to poor household economic conditions.

Most importantly, no access to flushed toilet is also another significant factor to increase probability of Jaundice transmission compared to the households using flushed clean toilets. All other things being equal, no access to a flushed toilet for the daily use increases the odds of Jaundice prevalence by 2.05 (95\% C.I. $=1.227$ 3.430). However, drinking boiled water and better management of solid wastes released from home, especially from the kitchen, can reduce the probability chance of Jaundice prevalence, compared to those not caring these sanitary activities. With high precision and accuracy, for a unit increase in the use of boiled water at household of local people, the odds of Jaundice prevalence probability is 2.101 (95\% C.I. $=1.206-3.661)$.

Table 2: Probit regression results

\begin{tabular}{llll}
\hline Variables & Coefficients (Model I) & Coefficients (Model II) & Odd ratios \\
\hline Warmer winter & $-0.383^{* * *}(0.136)$ & $-0.317^{*}(0.173)$ & $0.600^{*}$ \\
Untimely rain & $0.276^{*}(0.155)$ & $0.395^{* *}(0.166)$ & $1.972^{* *}$ \\
Heat wave & $0.541^{*}(0.283)$ & $0.308(0.292)$ & 1.721 \\
Family Size & & $-0.048^{* * *}(0.019)$ & $1.083^{* * *}$ \\
Agriculture as main source of income & & $-0.187(0.144)$ & 0.724 \\
Non-flushed toilet type & & $0.397 * *(0.150)$ & $2.052^{* * *}$ \\
Veterinary Centers & & $0.010(0.016)$ & 1.017 \\
Membership in microfinance & & $0.260^{*}(0.160)$ & $1.559^{*}$ \\
Distance to motorway & & $0.022(0.020)$ & 1.037 \\
Distance to health post & & $-0.013(0.035)$ & 0.975 \\
Management of solid wastes & & $-0.455^{* * *}(0.173)$ & $0.475^{* * *}$ \\
Boiled water preference & $-0.438^{* * *}(0.169)$ & $2.101^{* * *}$ \\
& $\mathrm{R}^{2}=0.037$ & $\mathrm{R}^{2}=0.107$ & $\mathrm{R}^{2}=0.108$ \\
\hline
\end{tabular}

$* * *=\mathrm{p}<0.01, * *=\mathrm{p}<0.05, *=\mathrm{p}<0.1$

Number in the parenthesis $=$ Standard Error

Source: Field Survey, 2018 


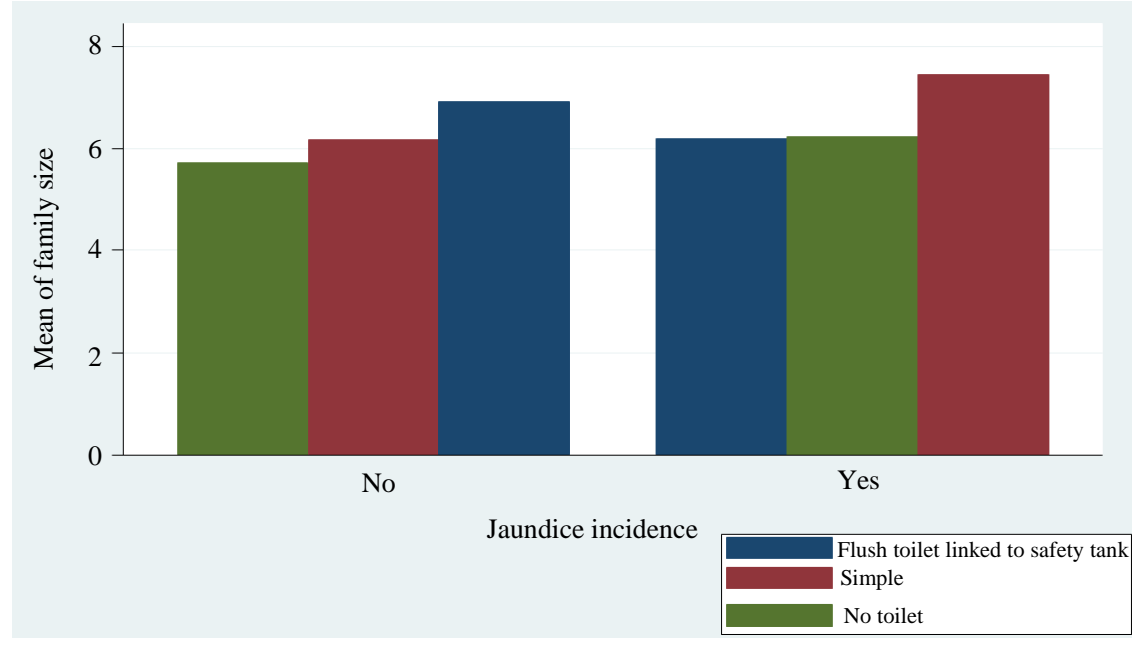

Fig. 1: Jaundice cases by toilet type categorized as per mean family size

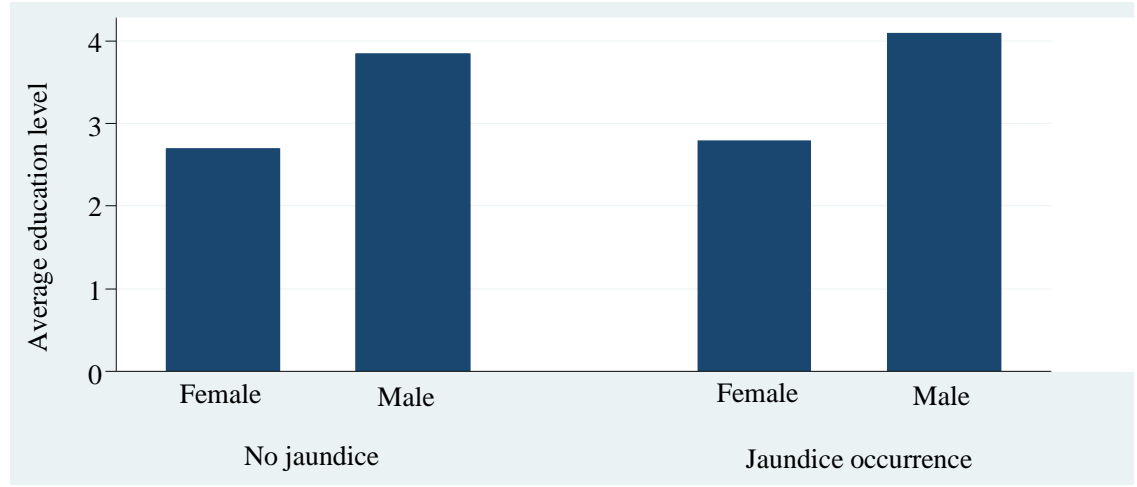

Fig. 2: Jaundice cases by gender's average education level

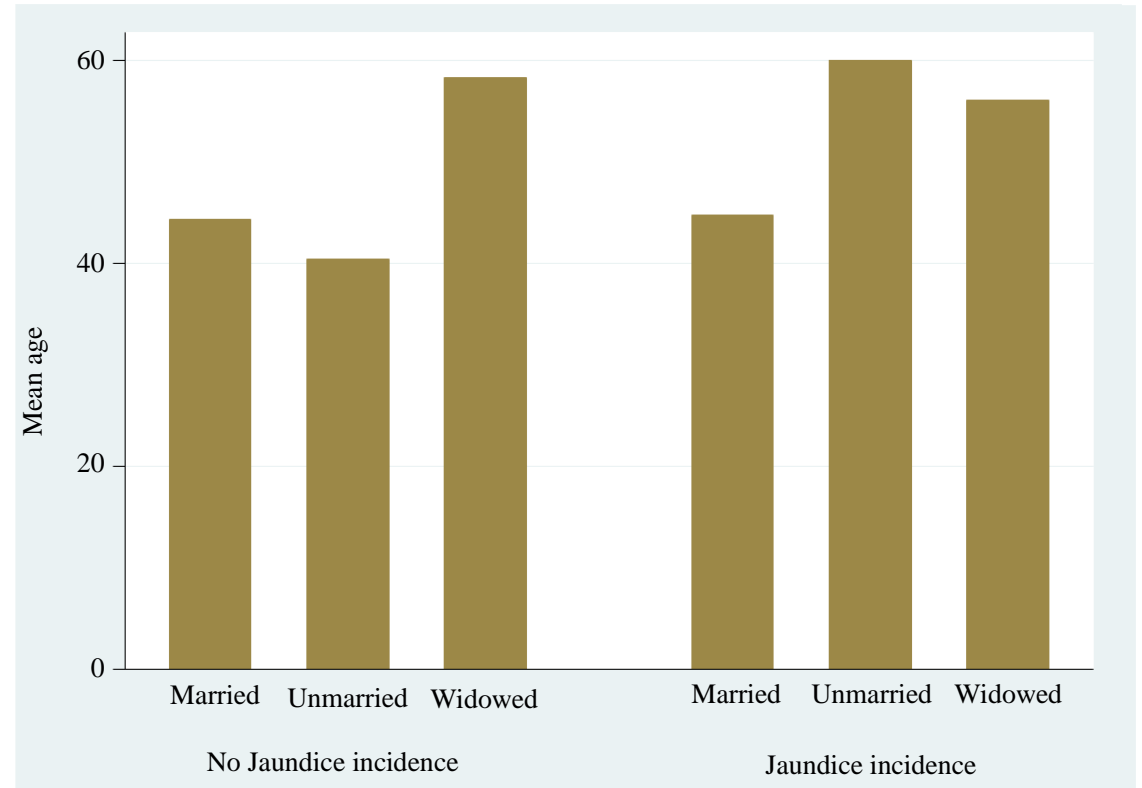

Fig. 3: Jaundice cases by marital status as perage 


\section{Discussion}

The study produced evidences on effects of environmental changes and household attributes on prevalence of Jaundice in Western Nepal. It might be representative for the developing countries facing severity of climate extremes and Jaundice prevalence in rural areas. Increasing trend of Jaundice occurrence as seen in annual unpublished government report of Government of Nepal along with evidenced environmental changes give alarms to the increasing disease burden of water communicable disease in western Nepal.

Warmer winter is unexpectedly seemed to lower the Jaundice prevalence. This might be logical to say that extreme cold in winter is not favorable for the human body by its warm blooded in nature, leading to disease exacerbation and severity particularly among rural poor. Further, warmer winter might aid to reduce the neonatal Jaundice cases because exposure of child in morning sun rays with mustard oil massage is common practice in community for the control of neonatal Jaundice and other diseases. Similarly, sporadic rain attributes in water contaminations through pathogens and abiotic mixtures and the water contamination is the major cause of Jaundice occurrence (Raguraman et al., 2017). Similarly, water contamination exacerbates the condition of Hepatitis-B infection (Ochwoto et al., 2016).

Heat waves are expectedly found as an environmental factor to increase Jaundice occurrence in western Nepal. This result is consistent with a study conducted in Bangladesh explaining the increasing hot air movement with increasing temperature results the exacerbation of water communicable diseases including Jaundice (Kabir et al., 2017). On the other hand, a study based on India also consistently concluded that movement of hot air promotes the transmission of infectious diseases like Jaundice (Raguraman et al., 2017).

With the family size, in general understanding, Jaundice is positively associated for the reason that chance of transmission of disease among many members is higher than few members in the family. It could be obvious in western rural Nepal where by observation family size seems bigger than the national average but not cared with the daily essentials. Besides, preference over boiled water keeps the personal health protected from water communicable diseases in some extent. Drinking safe water saves immune system alert to handle the entry of infectious disease during pregnancy as well (Cohen et al., 1972).

Distant kitchen from solid waste collection can reduce Jaundice. A well management of solid waste released from kitchen contributes to reducing Jaundice. Potential evidence (Ochwoto et al., 2016) exactly matched with expectation that non-flushed or simple type of single toilet used by all the family members seemed to get high chance of Jaundice prevalence for the reason that this type of toilet could create scope to transmit the disease vectors from diseased to non-diseased person due to the lack of adequate cleanliness with toilet cleaner.

To discuss with the limitations of the study; firstly, this study has rationally ignored some major environmental components such as improper land use and extreme weather events etc., which might yield potential influence over Jaundice. Secondly, this study is based on response of community people, leaving any experimental evidence, covering only some areas of western Nepal applying crosssectional survey-based study. Though this study poses some pitfalls, needy and urgent evidence on inevitable effects of environmental changes on Jaundice prevalence is produced in Nepal where such information is scanty. As Jaundice seems sensitive to temperature, trend of yearly increase in temperature might result into high level of cost of illness in Nepal (Paudel, 2018b).

Based on the above discussion over the Jaundice occurrence attributed by the different factors, community people are also quite knowledgeable about the environmental changes and household attributes. With the policy issues, this is the high time to take proper action over the protection of environment and adaptation strategy linked with infectious diseases and the health of the ecosystem under the natural resources such as soil, water that may results in unprecedented levels of disease emergence which potentially cause severe future impacts on human health. Based on the evidence from this research, Nepal government along with other global cohorts should implement urgent rural programs for the protection of environment that has negative effect on human health.

\section{Conclusion}

This research explored the major household and environmental factors encouraging Jaundice incidence in western Nepal by the use of probit model and marginal effects. This research developed a set of evidences that winter temperature, heat waves and untimely rain are the major environmental factors to be addressed by adaptation strategy for the sake of preventing people from Jaundice. Besides, importantly, control of family size, proper management of solid waste from kitchen, promoting in the use of flushed toilet at household level and promotion on habit of drinking boiled water are urgent need to reduce Jaundice cases in western Nepal along with the environmental protection and adaptation strategy of government and other global agencies working in environmental health issues.

\section{Data Availability}

The datasets analyzed during the current study are available from the corresponding author on reasonable request. 


\section{Acknowledgment}

The authors whole-heartedly acknowledge comments and suggestions from Faculty of Tribhuvan University; Bimala Baral - Co-worker in Research and University Grant Commission Nepal.

\section{Funding}

This research was conducted without any external funding.

\section{Author's Contributions}

Uttam Paudel: Prepared the manuscript.

Krishna Prasad Pant: Revised it and both the authors approved the manuscript.

\section{Conflict of Interest}

The authors declare that there is no any conflict of interest.

\section{References}

Azavedo, J.C.S., A. Drumm, C. Jupin, M. Parant and J.E. Alouf et al., 1988. Induction of tumour necrosis factor by staphylococcal toxic shock toxin. FEMS Microbiol. Lett., 47: 69-74.

DOI: 10.1111/j.1574-6968.1988.tb02493.x

Chen, C.F., M.C. Hsu, C.H. Shen, C.L. Wang and S.C. Chang et al., 2011. Influence of breast-feeding on weight loss, Jaundice and waste elimination in neonates. Pediatric Neonatol., 52: 85-92.

DOI: 10.1016/J.PEDNEO.2011.02.010

Chen, C.W., 1999. Political socialization and the cultivation of democratic citizens in Taiwan: A comparative study of the political attitudes and values of junior and senior high, junior college and college students. Issu. Stud., 35: 36-79.

Cohen, L., C. Lewis and I.M. Arias, 1972. Pregnancy, oral contraceptives and chronic familial jaundice with predominantly conjugated hyperbilirubinemia (Dubin-Johnson Syndrome). Gastroenterology, 62: 1182-1190. DOI: 10.1016/S0016-5085(72)80087-8

Dayrit, J.F., L. Bintanjoyo, L.K. Andersen and M.D.P. Davis, 2018. Impact of climate change on dermatological conditions related to flooding: update from the international society of dermatology climate change committee. Int. J. Dermatol., 57: 901-910. DOI: 10.1111/ijd.13901

Drew, J.H., J. Barrie, I. Horacek and W.H. Kitchen, 1978. Factors influencing Jaundice in immigrant Greek infants. Archives Dis. Childhood, 53: 49-52. DOI: $10.1136 /$ adc.53.1.49
Kabir, R. and S.M.Y. Arafat, 2018. Public health perspectives due to climate change in Bangladesh. Birat J. Health Sci., 2: 316-317. DOI: 10.3126/bjhs.v2i3.18956

Kabir, R., H.A.T. Khan, E. Ball, H.T.A. Khan and E. Ball et al., 2017. Climate change impact: The experience of the coastal areas of Bangladesh affected by cyclones Sidr and Aila. J. Environ. Public Health, 2: 316-317. DOI: 10.1155/2016/9654753

Kumar, K.P.S. and D. Bhowmik, 2011. Jaundice-review of clinical features, differential diagnosis and remedies. Pharma Res., 4: 241-252.

Mitra, A., S. Wajih and B. Singh, 2015. Wheezing ecosystems, livelihood services and climate change resilience in Uttar Pradesh. Asian Cities Climate Resilience.

Ochwoto, M., J.H. Kimotho, J. Oyugi, F. Okoth and H. Kioko et al., 2016. Hepatitis B infection is highly prevalent among patients presenting with Jaundice in Kenya. BMC Infec. Dis., 16: 1-14. DOI: $10.1186 / \mathrm{s} 12879-016-1409-2$

Paudel, U. and K.P. Pant, 2018. Environmental determinants of disease prevalence in rural Western Nepal. J. Environ. Protect., 9: 1221-1236. DOI: $10.4236 /$ jep.2018.912077

Paudel, U., 2018a. A review of effects of environmental change on human health. Am. J. Environ. Sci., 14: 95-109. DOI: 10.3844/ajessp.2018.95.1092018

Paudel, U., 2018b. Environment disease relationship and its impact on household economy. Biomed. J. Scientific Tech. Res. DOI: 10.26717/BJSTR.2018.10.001927

Paudel, U., S.R. Adhikari and K.P. Pant, 2020. Economics of environmental effects on health: A methodological review based on epidemiological information. Environ. Sustainability Indicators. DOI: $10.1016 / J . I N D I C .2020 .100020$

Paudel. U., S.R. Adhikari and K.P. Pant, 2018. Effects of environmental change on human health: A descriptive economic analysis. Am. J. Environ. Sci., 14: 274-287. DOI: 10.3844/ajessp.2018.274.287

Pires, B., J.V. Magalhaes and P.D. Gupta, 2016. Heat waves in non-conventional areas, Climate change and disease load: A review. J. Cell Tiss. Res., 16: 5705-5711.

Raguraman, S., R. Selvakumar and R. Sasikumarm, 2017. A statistical thinking on impact of climate change in health sciences. Int. J. Statist. Syst., 12: 395-406.

Slusher, T.M., T.G. Zamora, D. Appiah, J.U. Stanke and M.A. Strand et al., 2017. Burden of severe neonatal Jaundice: A systematic review and meta-analysis. BMJ Paediatrics Open. 